\title{
Stage IIIA2
}

National Cancer Institute

\section{Source}

National Cancer Institute. Stage IIIA2. NCI Thesaurus. Code C139981.

A stage term that applies to ovarian and fallopian tube carcinoma and indicates the presence of microscopic extrapelvic (above the pelvic brim) peritoneal involvement with or without positive retroperitoneal lymph nodes. There is no distant metastasis. (adapted from AJCC 8th Ed.) 